\title{
Decoupling emergence and reduction in physics
}

\author{
Karen Crowther \\ Department of Philosophy \\ University of Pittsburgh
}

\begin{abstract}
An effective theory in physics is one that is supposed to apply only at a given length (or energy) scale; the framework of effective field theory (EFT) describes a 'tower' of theories each applying at different length scales, where each 'level' up is a shorter-scale theory. Owing to subtlety regarding the use and necessity of EFTs, a conception of emergence defined in terms of reduction is irrelevant. I present a case for decoupling emergence and reduction in the philosophy of physics. This paper develops a positive conception of emergence, based on the novelty and autonomy of the 'levels', by considering physical examples, involving critical phenomena, the renormalisation group, and symmetry breaking. This positive conception of emergence is related to underdetermination and universality, but, I argue, is preferable to other accounts of emergence in physics that rely on universality.
\end{abstract}

\section{Introduction}

In philosophy, the vast literature on emergence is almost matched in size by the wildly diverse range of uses of the term 'emergence' itself. As Silberstein (2012) states in his recent review of three large edited collections on emergence: it is absurd to argue that any particular conception of emergence is superior across the board. Instead, philosophers should embrace the need for pluralism, as different cases require different conceptions of emergence. My aim in this paper is to understand the conception of emergence appropriate to physical cases where systems are described by different theories depending on the length (or energy) scales under consideration.

Such a project requires one to embark with a rather specific grade of idea: either too vague or too strong a prior conception of emergence and we risk finding nothing of interest. On the one hand, we need to capture enough of what philosophers take 
to be important in defining a conception of emergence for the concept to still be understandable as emergence, but, on the other hand, we do not want to be so tied to a prior conception of emergence that we would be able to learn nothing significant from the physical examples (other than to what extent they may be said to embody the conception of emergence we begin with). This difficulty is compounded, of course, by the fact that there is no single general or 'best' definition of emergence that applies generally, but rather many different conceptions, each with its own advantages and domain of applicability.

Because my aim is to understand the physics, rather than develop an account of emergence for other areas of philosophy, I am here less concerned with conforming to some existing definitions (and with so alienating some other philosophers) than I am with elucidating the relations exemplified in the physical cases that I consider. The notion of emergence that I find by looking at these physical examples is one that differs from the standard philosophical accounts. In particular, it is a conception that is best not associated with the idea of reduction (in any sense) focusing on the ideas of deduction and derivability is not helpful when considering the physical examples. Yet, because emergence is typically so bound up with reduction, I am willing to relinquish the term 'emergence' if the reader feels more comfortable to think of the 'emergence-disassociated-from-reduction' relation as something other than emergence. Readers should feel free to view my suggestion as an interesting and significant relation in physics without feeling that it undermines their own preferred understanding of 'emergence'.

The first of the physical examples I consider is effective field theory (EFT), a framework that inspired Anderson (1972) in writing his well-known 'More is Different', and whose interpretation became even more controversial following Cao and Schweber's (1993) historical and philosophical exposition. ${ }^{1}$ An effective theory is one that effectively captures the physics relevant at a given length (or energy) scale (Georgi , 1993). In other words, it is a theory supposed only to be valid in a particular regime (though in fact it may or may not be valid for length scales outside of this regime). EFT refers to both the framework of EFT as well as the theories that are associated with it; in this paper, 'EFT' usually refers to the framework, while 'EFTs' refers to the theories themselves, and 'an EFT' denotes a particular effective field theory. The typical understanding of EFT is that it presents us with a 'tower of theories' ordered hierarchically according to energy scale (or inverse length scale), where high-energy (short-distance) theories are toward the 'top' and lower-energy (larger-distance) theories represent lower

\footnotetext{
(1992).

${ }^{1}$ See, e.g. Castellani (2002); Huggett and Weingard (1995); Hartmann (2001); Robinson
} 
'levels' in the tower. ${ }^{2}$ Each of the levels is (quasi-) autonomous, able to be studied independently of the one above it, yet the entities of any particular level are understood to be somehow constituted by those of the level above it. A crude example of the tower (where the levels are not, strictly speaking, 'theories' but whole disciplines) is: quantum gravity, particle physics, condensed matter physics, chemistry, biology. The levels are usually supposed to be linked by the relation of emergence, where the lower-energy theory is said to emerge from the higher-energy theory above it.

Although the idea of emergence in EFT has attracted much attention and been the source of much controversy, the relation between 'levels' remains opaque. Indeed, philosophers have propounded views that apparently contradict one another. One of the problems contributing to the confusion, I argue, relates to the general preoccupation of philosophers with the idea of ontological emergence, and the corresponding focus on the idea of a failure in principle of reduction (derivability or deduction). A particular subtlety regarding the necessity and role of EFT, however, threatens the relevance of any conception of emergence that is related to reduction. Also, focusing on issues of reduction and derivability may cause us to overlook the important aspects of the actual physics (i.e. EFT as it is used in practice). Instead, I find the better conception of emergence to be one based simply on the novelty and autonomy of the low-energy level compared to the highenergy level above it (to which it is linked by EFT methods). The ideas of novelty and autonomy also usefully apply to the physical examples I consider, involving symmetry-breaking and the renormalisation group. The intended senses of novelty and autonomy are defined in $\S 4$ but are developed throughout the paper. As I argue, there are several ways in which the novelty and autonomy may come about; in this paper I am especially interested in underdetermination and universality as leading to a sense of autonomy.

A quick example should give some feel for what is meant. Consider the BCS theory of superconductivity as an example, typical of EFT, where the low-energy theory severely underdetermines the high-energy physics. The BCS theory is an approximation which ignores most of the mutual interactions of the electrons of the system and focuses only on a particular interaction due to phonon exchange. ${ }^{3}$

\footnotetext{
${ }^{2}$ Here, I'm following the convention used in the physics literature, where the shorter-distance theory is 'top' and the larger-distance theory that emerges from it is 'bottom', because these theories are usually defined in terms of energy, rather than length, scales (and energy scale is inverse to the length scale). Please note that this is the contrary to how the terms are usually used in the philosophy literature.

${ }^{3}$ Phonons, a type of quasi-particle, are the excitations of quantised modes of vibration (i.e. sound particles). Importantly, they are collective excitations which exist only at low energies,
} 
In spite of this, it works surprisingly well in many situations, with its predictions often in agreement with experiment to within several percent. The success of the BCS theory can be explained by the framework of EFT: it can be shown that only the specific interactions used by the BCS theory are relevant at low energies. All the rest of the interactions - important in the high-energy theory - are suppressed at low energies by powers of a small energy ratio (Burgess , 2004, p. 9). This example demonstrates how little the low-energy physics (of interest) depends on the high-energy (micro-) physics: most of the high-energy interactions can simply be ignored. Given the low-energy theory alone, it would be impossible to come up with the high-energy theory without the aid of experimental results or some other external source of input. In this example, we can thus say that the low-energy physics is relatively autonomous from the high-energy theory.

The BCS theory also furnishes an example of universality: that is, behaviour exhibited by many different systems (essentially similar to the idea of multiplerealisability, though with an additional requirement that will be explained in §5). The example is the success of the BCS theory as applied to the Josephson Effect, which is used to determine the value of the fine structure constant in precision tests of quantum electrodynamics (QED). In this case, the agreement with experiment is in matter of a few parts per million! Remarkably, this success is not due to any of the details of the BCS model except for one important symmetry-breaking pattern it predicts. As Burgess (2004) explains, the Josephson prediction follows on general grounds from the low-energy limit of a broken gauge symmetry: any theory with the same low-energy symmetry-breaking pattern shares the same predictions. The fact that different high-energy systems exhibit the same low-energy behaviour means that the low-energy physics in question is autonomous from the high-energy (micro-) theory.

The paper proceeds as follows. I begin by outlining (in $\S 2$ ) the formalism of EFT and the idea of emergence it suggests. I then compare this to the more standard conceptions of emergence in philosophy which, I claim, are not usefully applied to real physical cases - the reason being that conceptions of emergence in philosophy are typically associated with a failure of reduction (in some sense). This focus on reduction is amplified by the general compulsion to divide and classify cases of emergence as being either ontological or epistemological. I will argue that this is neither helpful nor interesting when considering the physics, and that we do better to take emergence as unconcerned with reduction. However, since, as stated above,

and do not appear in the high-energy description of the system. In other words, the low-energy theory of phonons is novel compared to the high-energy theory (the idea of novelty will be explained in $\S 4$ ). 
there is no single best definition of emergence, the discussion in $\S 3$ is only intended to motivate a positive account of emergence in the philosophy of physics, rather than to deny that there may be conceptions of emergence linked to reduction. My point here is in line with Butterfield (2011a,b) who demonstrates that there is no necessary connection between emergence and reduction. ${ }^{4}$ The positive conception of emergence I suggest - novelty and autonomy — is presented in $\S 4$.

After explaining how these ideas feature in EFT, I turn to the idea of universality as a basis for a conception of autonomy $(\S 5)$. Universality is a more specific form of underdetermination in EFT, representing cases in physics where we have actual examples of different high energy systems with the same low energy physics (in the more general underdetermination cases, there is the possibility of such systems, but they may not be known to us). There are two recent accounts of emergence that rely on universality; Batterman (2011) is concerned with critical phenomena (second-order phase transitions) and fixed points in the renormalisation group (RG) flow (which I outline in §5.1) and argues that mathematical divergences are essential to understanding emergence. Morrison (2012) appeals to the example of superconductors ( $(5.2)$, and emphasises the role of symmetry-breaking as a mechanism for universal phenomena and an explanation of emergence. I argue, in $\S 6$, that both these accounts suffer for being artificially restrictive (applying only to specific EFTs, rather than all EFTs), as well as for their reliance upon a definition of emergence framed in terms of a failure of reduction. My positive conception of emergence, which is developed by considering EFT as it applies more generally (i.e. including in non-critical cases), is preferable for its benefits of fitting more naturally with the physical examples, as well as avoiding the problems associated with the focus on reduction.

\section{The framework of effective field theory}

As will be demonstrated in the rest of this paper, the framework of EFT applies in many different areas of physics, particularly condensed matter physics. My introduction in this section, however, is framed in terms of how EFT is used in quantum field theory (QFT). Prior to the application of RG techniques and the idea of EFT, QFT suffered from conceptual difficulties relating to the need to remove divergences produced by perturbative calculations in the theory before any physical predictions could be obtained from it. The framework of EFT and the philosophy that accompanies it resolves these difficulties by holding that the

\footnotetext{
${ }^{4}$ I discuss Butterfield's (2011a,b) conception of emergence, and how my account compares to it, in $\S 3.1$.
} 
divergences are a consequence of mistakenly assuming that our theories are valid to arbitrarily high energies.

Traditionally, the divergences are tamed through a two-step process of renormalisation. Firstly, the theory is 'regularised' through the introduction of a cutoff energy of some finite value, $\Lambda$, which serves to remove the high-energy states from the theory. Secondly, the theory is altered in order to compensate for the removal of the high-energy interactions, and this is done by redefining the parameters (i.e. charges, masses and other coupling constants) that feature in the theory. The original 'bare' parameters are replaced by physical 'renormalised' parameters that absorb the divergences of the original theory. Once all calculations had been performed, the traditional procedure is to remove the artificially-imposed cutoff, $\Lambda \rightarrow \infty$, and restore a continuum theory. In the 'new conceptualisation' of renormalisation, thanks to the $\mathrm{RG}$, however, the cutoff is no longer a mathematical artifice to be viewed with suspicion and promptly removed at the end of the day, but is instead retained as being of physical significance. The cutoff is held to represent the upper edge of the theory's domain of validity - it is a high energy (equivalently, heavy mass) scale that serves to demarcate the low-energy physics (as dealt with by the theory) from the high-energy physics (not dealt with by the theory).

A change in the value of the cutoff-for instance, rescaling it to a lower energy, $\Lambda_{0} \rightarrow \Lambda(s)=s \Lambda_{0}$ - can thus be compensated for by a rescaling of the parameters that feature in the theory. A smooth change in the energy scale will define a trajectory in parameter space; for instance, for one parameter (or coupling constant) $C$, a trajectory $C=C(s)$ is defined as $\Lambda(s)$ varies. The parameters which feature in the theory are thus said to 'run' or 'flow' as the energy scale changes. The RG equations describe how the parameters in the theory vary with the energy scale being probed. ${ }^{5}$ Generally, they are given as beta functions,

$$
\beta(C)=E \frac{\partial C}{\partial E}=\frac{\partial C}{\partial \ln C}
$$

where $E$ is the energy scale under consideration, and $C$ the coupling constant. For a set of coupling constants, $\left\{C_{k}\right\}$, we have,

$$
\left\{C_{k}^{\prime}\right\}=\beta\left(\left\{C_{k}\right\}\right)
$$

where the beta function is said to induce the flow of the set of coupling constants, $\left\{C_{k}\right\}$.

\footnotetext{
${ }^{5}$ For details see, e.g. Lepage (1989); Zee (2010) III.1.
} 
The RG equations reflect the idea that the effective values of the parameters in our theories are products of interactions, and change with the strength of these interactions.

It often happens that, as we move to larger observation-distance scales (i.e. decreasing the value of $\Lambda$ in the RG equations), the set of physical coupling constants, $C^{\prime}$, flow to a fixed point, at which further increase of $\Lambda$ produces no change in the value of $C^{\prime}$. When the fixed point is reached in this way, by decreasing the energy scale, it is known as an infrared (IR) fixed point. This will be important when discussing the conception of emergence based on critical phenomena, in $§ 5.1$. On the other hand, an ultraviolet (UV) fixed point is one that is approached as the value of $\Lambda$ is increased.

The RG enables low-energy theories to encode the low-energy effects of the highenergy interactions, without the theories explicitly acknowledging the existence of the high-energy interactions. ${ }^{6}$ This is how we are able to speak of low-energy phenomena independently of the underlying high-energy physics. As we consider different energies, the contributions of the various interactions responsible for the values of the parameters in our theory are accounted for by the RG equations, so long as we are working at energies well below the cutoff, $\Lambda$. Moving to higher energies, approaching the cutoff, however, the RG equations start to show signs of a breakdown. This is taken as a hint that unknown physics is coming into play; the effects of some high-energy states are no longer able to be incorporated by a reparametrisation of the currently employed terms. A new QFT must be constructed whose Lagrangian contains extra terms in order to explicitly account for the new high-energy interactions. This theory will then, in turn, be applicable only at energies well below some new cutoff, of energy greater than that of the original theory.

The core idea of EFT is that the physics of some system at energy $E$ can be described in terms of a power series expansion in small dimensionless parameters, $E / \Lambda_{i}$, where $\Lambda_{i}$ are various physical scales (i.e. heavy particle masses) involved in the system which are larger than $E$. The effective Lagrangian, $L$, is written as a linear combination of all the operators, $O_{i}$, which are allowed by the symmetry constraints of the theory,

$$
L=\sum_{i} c_{i} O_{i}
$$

\footnotetext{
${ }^{6}$ In 'traditional QFT', recall, the parameters of the theory are replaced with renormalised parameters that include counter-terms to cancel the divergences. These new parameters compensate for the neglect of the high-energy states, and so 'encode' the effects of the high-energy interactions, whatever they may be.
} 
where $c_{i}$ are coupling constants, whose size depends on a heavy energy scale $\Lambda$, and the dimension, $d_{i}$ (of the $i$ th operator, $O_{i}$ ) compared to the dimension of spacetime (here taken to be 4) so that: $c_{i} \sim 1 / \Lambda^{d_{i}-4}$. This can be more clearly written by defining dimensionless coupling constants: $\Lambda_{i}=c_{i} \Lambda^{d_{i}-4}$, so that the order of the $i$ th term in $(3)$ is $\Lambda_{i}(E / \Lambda)^{d_{i}-4}$.

The operators fall into three categories, based on their dimension compared with the dimension of spacetime. ${ }^{7}$ Operators with $d_{i}<4$ have positive-dimensional coefficients and are called relevant, because they are relevant at low energies, but get smaller as $E \rightarrow \Lambda$. Irrelevant operators have $d_{i}>4$ and thus negative-dimensional coefficients. So, at low-energies these terms are suppressed by positive powers of $(E / \Lambda)$ (which is a small number, since $E<<\Lambda$ ). They become important, however, as $E \rightarrow \Lambda$. Finally, operators with $d_{i}=4$ and dimensionless couplings are called marginal, and are constant at low and high-energy (marginal terms, however, will almost always have their dimension altered once quantum corrections are taken into account and, hence, they become either relevant or irrelevant). Typically the expansion (3) will contain only a finite number of marginal and relevant operators. It can, however, contain arbitrarily many irrelevant operators. ${ }^{8}$

The EFT approach is premised on there being a large gap between the energy being studied, $E$, and the energy scale at which new physics appears, with the latter characterised by a heavy mass, $\Lambda$, representing the theory's cutoff. In the regime where $E<<\Lambda$, the irrelevant operators are suppressed by powers of $(E / \Lambda)$ in the expansion and the EFT behaves for all practical purposes as a renormalisable field theory, containing only a finite number of operators.

\subsection{Top-down and bottom-up}

There are two ways to approach EFT: either top-down, or bottom-up (with the terms bottom-up and top-down referring to relative positions and motion along the energy scale). The bottom-up approach is taken when there is a low-energy theory for the system being studied, but a higher-energy (shorter-scale) theory is sought: in other words, there is a theory with cutoff $\Lambda_{1}$, but a theory with cutoff $\Lambda_{2}>\Lambda_{1}$ is required. This approach involves probing accessible energies and constructing the theory in response to the particular experimental situation; typically, it requires

\footnotetext{
${ }^{7}$ In natural units, where the speed of light and the reduced Planck's constant are taken as equal to one, all quantities of length $L$, mass $M$ and time $T$ can be expressed (dimensionally) as a power of energy $E$, using dimensional analysis. This allows us to express the dimension of an operator in terms of length, where $E=L^{-1}$.

${ }^{8}$ This account is drawn from Pich (1998); Polchinski (1993).
} 
adding new terms to the theory in order to allow for unknown high-energy effects as they become important. ${ }^{9}$

The top-down approach is taken when the high-energy theory, with cutoff $\Lambda_{2}$ is known, but a low-energy theory, of cutoff $\Lambda_{1}$ where $\Lambda_{1}<\Lambda_{2}$ is required. In principle the new theory can be constructed in a totally systematic way. One method is by 'flowing' (rescaling) with the RG down to the energy scale $\Lambda_{1}$ (representing a particle of mass $M_{1}$ ) and using 'matching conditions' to ensure that the high-energy theory agrees with the low-energy theory in its experimental predictions at the 'boundary' defined by $M_{1}$. Another means is by 'integrating out' the high-energy interactions and expanding the resulting low-energy effective action in a sum of operators, as in (3). ${ }^{10}$ Either of these procedures, in principle, would result in a tower of theories, with each EFT framed in terms of the relevant degrees of freedom and describing a different 'level', defined by the energy scale of its high-energy boundary. Each of the theories in the tower would be effectively decoupled from the one beneath it. ${ }^{11}$

In practice, however, arriving at a useful low-energy theory is not always so simple. Although there are cases where the systematic 'recipe' for constructing an EFT is able to be applied straightforwardly, there are other cases where it applies with varying degrees of difficulty. The crucial first step is identifying the appropriate low-energy degrees of freedom for the system being studied. This is often a non-trivial task, because the low-energy degrees of freedom may be a far cry from those which feature in the high-energy theory. For example, the high-energy description of a condensed matter system may be framed in terms of electrons and nuclei, while the low-energy physics (such as superfluidity and superconductivity) is best described by a theory of quasi-particles (phonons), which arise as collective excitations and are simply not manifest at short distances. Typically, in the difficult cases, an external means of guidance is required in order to identify the low-energy degrees of freedom when given only the high-energy description; this may be the results of experiment or some other source of inspiration. EFT often applies less systematically and more like an art, with theoreticians pulling the necessary approximation techniques from the toolbox of EFT.

\footnotetext{
${ }^{9} \mathrm{~A}$ concise, non-technical overview is provided in Hartmann (2001).

${ }^{10}$ These two different methods are known as the continuum EFT and Wilsonian EFT approach, respectively, and may differ as to their interpretation (Bain , 2013a).

${ }^{11}$ In Wilsonian EFT, this idea is formalised in the decoupling theorem of Appelquist and Carazzone (1975).
} 
A tower of theories can be constructed in this manner, where the theories represent different 'levels' according to the energy scale at which they are applicable, and each is able to be studied (for the most part) independently of the level 'above' it at higher energy (again, I emphasise that this way of speaking runs in the opposite direction to the usual way, where we are used to saying that the "level above" represents the coarser-grained theory, rather than the finer-grained one).

\subsection{Understanding emergence in EFT}

The idea of a tower of theories, where each is said to 'emerge' from the one above it on the energy scale (or, equivalently, the theory underlying it, 'beneath' on length scale) is a much debated one. The controversy stems not only from the link to Anderson's (1972) claims regarding fundamentality in 'More is Different', or Cao and Schweber's (1993) radical suggestion that the tower construction implies a pluralism of ontology, but also from the idea of 'emergence' that is supposed (or not supposed) to be manifest in EFT. In addition, there has been some 'cross-talk' between (i) authors who focus on the EFT framework as it applies in principle, and (ii) those who speak of it as it is actually used in physics, resulting in a number of conflicting claims in the literature. For instance, while Castellani (2002) claims that EFT does not imply antireductionism, given that there is in principle a systematic means of arriving at the low-energy physics given the high-energy theory, Bain (2013a,b) is concerned with EFT as it applies in practice, stressing the role of approximations in building an EFT and the (often non-trivial) task of first identifying the relevant degrees of freedom.

Bain's (2013b) account of emergence in EFT emphasises the fact that the highenergy theory and the low-energy EFT are formally distinct and derivationally independent. Being formally distinct means that the theories are represented by different Lagrangian densities, which describe different dynamical variables; specification of the equations of motion for the high-energy theory (together with pertinent boundary conditions) will fail to specify solutions to the equations of motion of the EFT. The claim of derivational independence is based not only on the formal distinctness of the theories, but the fact that, in practice, the use of approximations and/or other means of external guidance is necessary in many cases in order to construct the low-energy EFT, even given the high-energy theory. This suggests, Bain states, that it is unlikely that the steps involved in constructing an EFT could be understood, or reformulated, as a derivation. Bain then goes on to examine how this derivational independence relates to different forms of autonomy -including reductive autonomy, predictive autonomy, causal autonomy, and explanatory autonomy - taking "emergence" to be constituted by any, or all, of these. 
While Bain $(2013 \mathrm{a}, \mathrm{b})$ is correct in his assessments regarding the top-down construction of EFTs, the picture he presents stands in tension with that of Castellani (2002): on the one hand, there is, in principle, a 'systematic' method of deriving a low-energy theory given only the high-energy physics, but, on the other hand, typically the low-energy theory is derivationally independent of the high-energy theory. Reconciling these two accounts requires a bit of work. Consider the example of quantum chromodynamics (QCD), which is a high-energy theory of quarks and gluons: particles believed to make up hadrons, such as protons and neutrons, at low energy (large distances). We cannot solve QCD at low energies. But, researchers are slowly pushing forward in the ab initio derivation of the hadron masses using numerical methods (i.e. tedious approximation techniques). This means that QCD does, in principle, contain all the information required in order to make low-energy predictions. Nevertheless, it seems impossible to derive a theory of hadrons - an EFT - from QCD only. External input is required. It is important to note, too, that EFT (which in this case amounts especially to chiral perturbation theory) is not the only means of extracting low-energy results from QCD. ${ }^{12}$

In other words, although often extremely difficult in practice, it is possible in principle to obtain low-energy quantitative results from the high-energy theory. In the typical situation where we cannot in practice obtain results directly from the high-energy theory (presumably because of our own fallibility), the framework of EFT is just one of several different techniques for doing so. For this reason we should be wary of speaking of the framework as though it is strictly necessary for obtaining quantitative low-energy predictions - there are other ways of getting numbers out of a theory. The framework of EFT, however, is necessary in a more subtle sense. An effective, low-energy theory is the only means of properly describing the low-energy behaviour of a system. EFTs are formulated in terms of the appropriate degrees of freedom for the energy being studied, and are necessary for imparting an understanding of the low-energy physics. Because the low-energy degrees of freedom do not exist at higher energy, the high-energy theory is unable to present the relevant low-energy physics.

Thus, we can distinguish between an EFT's role in enabling quantitative predictions in the low-energy regime - a role which, in principle, could be fulfilled by the high-energy theory - and its role in appropriately describing the behaviour of a system at low energy, and thereby facilitating an understanding of the low-energy

\footnotetext{
${ }^{12}$ Other methods include the lattice approximation (Bazavov et al. , 2010; Callaway and Rahman , 1983, 1982; Dürr et al. , 2008), QCD sum rules (Shifman , 1998) and the NambuJona-Lasinino model (Nambu and Jona-Lasinio, 1961a,b).
} 
physics - a role which could not be fulfilled by the high-energy theory. Grasping this subtlety means realising that the focus on derivability as a basis for emergence in EFT is a mistake. It is irrelevant to the relations between the 'levels' that are actually interesting and important. This is motivation to step away from concerns regarding reduction, to leave aside (for present purposes) the metaphysical debates and concepts emphasised by the philosophy of mind, and instead find a conception of emergence that is inspired by, and applicable to, other problems in physics. ${ }^{13}$

In developing a conception of emergence in EFT, its non-reliance upon a conception of reduction is an asset. When we possess only an effective theory, we have very little indication of what the high-energy theory is that it emerges from. Such is the case for the QFTs of the standard model of particle physics, which are understood as EFTs of some unknown high-energy physics, as well as general relativity, which is understood as the EFT of quantum gravity (a theory we do not possess). Understanding the relationships between high-energy physics and lowenergy physics in these cases cannot involve a strong sense of reduction (we have no theory to reduce to) but we still have an idea of emergence - the low-energy physics is novel and autonomous from the high-energy physics. The framework of EFT tells us that the high-energy theory could have any structure whose low-energy features are not forbidden by the symmetries and parameters of our low-energy theories - and, in turn, the details of this theory could be anything consistent with these possible structures. In other words, the low-energy physics severely underdetermines the high-energy theory. So, the fact that the account of emergence that I develop here is not associated with a definite sense of reduction makes it more useful than it would be otherwise - it enables us to understand emergence even in cases at the very edge of physics (for instance, it can be used in exploring the idea of the emergence of spacetime, and other structures in GR, from quantum gravity).

\section{Decoupling emergence and reduction}

There exists a multitude of definitions of emergence in philosophy. Nevertheless, a very vague, general, or intuitive definition of emergence may be taken as com-

\footnotetext{
${ }^{13}$ Of course, I do not mean to imply that there is no possible conception of reduction whose failure could be used to formulate a definition of emergence in these cases - many authors have considered specific examples of EFTs and have gone ahead to formulate such definitions (I consider some of these in $\S 6$ ). Similarly, I do not mean to suggest that the account of emergence that I present in this paper has no bearing on any possible conception of reduction; rather, my point is that it is uninteresting, for the purposes of understanding our physical theories and the relationships between them, to seek out such a conception. This point is argued for further in the next section.
} 
prising two claims. Bedau (1997, p. 375) puts them thus (although the labels 'Independence*' and 'Dependence*' are mine):

Dependence* Emergent phenomena are somehow dependent on, constituted by, generated by, underlying processes.

Independence* Emergent phenomena are somehow autonomous from underlying processes.

Typically, the first of these claims, 'Dependence*', is relatively uncontroversial, and it is the second claim that serves to distinguish different conceptions of emergence. In the vast majority of cases, 'Independence*' involves ascribing one or more of the following features to the emergent phenomenon (property or theory): irreducibility, unpredictability, causal independence, or unexplainability given its microphysical base. In other words, the claim that some phenomenon is emergent is usually understood as the claim that the phenomenon is in some sense not reducible to (i.e. deducible from) its base.

This leads us to the core distinction in the emergence literature. Ontological emergence is the thought that this failure of reduction (in whatever sense is meant) is a failure in principle: that there are "genuinely emergent" phenomena (properties, systems, theories, etc.). It is emergence in a strong sense. This stands in contrast to epistemological emergence, which is a failure of reduction in practice, meaning that the apparent emergence is (somehow) really only an artefact of our computational limitations. Epistemologically emergent phenomena are not thought to be 'genuinely emergent', but, for whatever reason, it is very difficult for us to explain, predict or derive them on the basis of their underlying system(s). This leads to epistemologically emergent properties being termed predictive or explanatory emergent properties.

Hence, the difficulty in articulating a conception of 'Independence*' that characterises emergence usually becomes the difficulty in articulating the conception of reduction that is not being exemplified. This is not automatically a helpful shift, because the use of the term 'reduction' is as ubiquitous and heterogeneous as 'emergence'. Taking this path, and choosing a particular definition of reduction also necessarily means closing the door on many other, incompatible, views, and thus ending up with a conception of emergence with restricted applicability and interest. ${ }^{14}$ Rather than attempt the long, exclusive - and possibly treacherous - path

\footnotetext{
${ }^{14}$ As an example of these difficulties, consider Butterfield and Isham (1999) who attempt to formulate an appropriate definition of reduction using the precise, intuitive idea of definitional extension, only to conclude that such a relation is, at once, both too strong and too weak to do the
} 
of seeking out a particular definition of reduction whose failure best characterises emergence, it is better, when considering the physical examples that I do in this paper, to focus on a positive definition of emergence, unconcerned with reduction. ${ }^{15}$ This positive definition is naturally suggested by the physics, and has the advantages of being straightforward and general.

The desire to think of emergence in terms of reduction is tied to the metaphysicians' fixation on ontological emergence, the need to sharply distinguish it from "merely" epistemological emergence. The question of how language relates to the world is one of the most difficult and well-debated ones in philosophy; the question of how our theories relate to the world is another. While these questions certainly have implications for the philosophy of physics, we can do philosophy of physics without tangling with them. Similarly, if we are doing philosophy of physics because we are interested in understanding physics and its philosophical foundations, rather than as a means of arriving at metaphysical or ontological conclusions, then we are not automatically compelled to engage with these very interesting, but distinct, areas of philosophy. Taking emergence as a failure of reduction or derivation forces the focus of inquiry onto the question of what counts as a reduction or derivation, and distracts from the lessons of the actual physics. Resisting the desire to define emergence in terms of reduction means looking at physics as it is actually practised rather than speculating about what we could do with our theories "in principle". It means developing an account true to the science rather than seeking to carry-over prior intuitions and concepts from other branches of philosophy.

\subsection{A varied landscape where less is different}

Of course, I am not the only one to claim that emergence may be separated from reduction. Butterfield (2011a,b) and Butterfield and Bouatta (2012) have written a series of papers in which the independence of emergence and reduction is demonstrated, using an assortment of physical examples, including phase transitions. These show that we can have emergence with reduction, as well as emergence without reduction. Emergence is defined simply as novel and robust behaviour relative to some comparison class, and reduction is defined as deduction

job. Also, in the case of definitional extension, for example, we are required to adopt the syntactic conception of theories (treating a theory as a set of sentences closed under deduction), which means neglecting a very popular rival view of theories, viz. the semantic conception (according to which theories are classes of models), as well as excluding conceptions of emergence that hold between things other than theories.

${ }^{15}$ Again, however, see Footnote 13. 
aided by appropriate definitions or bridge principles (i.e. Nagelian reduction). ${ }^{16}$ I view Butterfield's results as evidence that we should step aside from defining emergence in terms of reduction, and finally stop debating the issue of "emergence versus reduction".

However, apparently not everyone who considers the demonstrated reconciliation of emergence and reduction in Butterfield (2011a,b) has the same response. Callender (2013), for instance, follows Butterfield in exploring the example of phase transitions, but is concerned with the claims of several authors who argue that, because reduction fails in some sense (between the thermodynamic description of the phenomena and the statistical mechanical one, owing, in some way, to the thermodynamic limit), phase transitions represent emergent phenomena. For each of these claims, Callender (2013) finds a different way in which reduction does not fail. So, while Butterfield argues that emergence and reduction are logically independent (i.e. we can have emergence with reduction, as well as emergence without reduction), Callender (2013, p.32) argues that there is no sense of emergence that poses a threat to "the reductionist program broadly construed". Callender's conclusion is revealing of philosophers' willingness to engage in the grand battle between 'emergentists' and 'reductionists', and the sad fact that any claim of emergence is often only of interest for its consequences regarding reduction. The point I want to make is that, if we are interested in understanding our physical theories and the relationships between them, we do not need to take up arms - we can explore emergence without having to engage with issues of reduction (even though there may be various ideas of reduction in the vicinity). Indeed, the shift in focus promises to enable us to see new questions in the philosophy of physics that we otherwise would overlook.

\section{A positive conception of emergence}

So far I have presented a case for disassociating emergence and reduction, and have suggested that we instead focus on the positive aspects of emergence. ${ }^{17} \mathrm{~A}$

\footnotetext{
${ }^{16}$ This conception of emergence is essentially similar to the one I propound here. As will be shown in $\S 4$ however, my account features an additional aspect (Dependence). Also, I go further than Butterfield in articulating the basis for novelty and autonomy (I do this using the ideas of underdetermination and universality, which are not part of Butterfield's (2011a,b) account); and, unlike Butterfield (2011a,b), my account here does not make reference to limits. Another important contrast is that my account is supposed to apply to EFTs in general, while Butterfield (2011a,b) considers only specific examples of EFTs.

${ }^{17}$ As stated, I do not want to argue that this is the best or only account of emergence, simply that it is the best account for the physical examples considered here. I do not want to put people off, so if you would rather not call this 'emergence', please call it something else instead (perhaps
} 
tentative schema is thus,

Dependence The low-energy theory is related to the high-energy theory via the physics of the RG and EFT techniques. Alternatively, if one is comfortable with the concept of supervenience, we can understand Dependence as involving supervenience ${ }^{18}$ : the system described by the low-energy (macro-) theory supervenes on that of the high-energy (micro-) theory. The claim is simply that two systems that are the same according to a particular highenergy theory (whatever the appropriate one may be for the system and energy under consideration), can be described as having the same physics (as one another) by the appropriate low-energy theory. ${ }^{19}$

Independence The low-energy physics is novel and autonomous with respect to the high-energy description.

The claim of 'Dependence' serves only to define the comparison class which is, in most cases, appropriate when discussing the physical examples. The RG demonstrates that there is a 'direction' in physics, where the low-energy physics depends, in some minimal sense, on the high-energy physics, but not vice-versa. Here, the 'high-energy' physics need not (and should not) be taken as an 'ultimate' highenergy description or 'final theory' describing some smallest or elementary entities, and, indeed, neither should it be taken to imply the existence (or non-existence) of such a theory. Instead, the high-energy theory may simply be understood as the 'next level up', bounded by $\Lambda_{2}>\Lambda_{1}$ where $\Lambda_{1}$ is the energy-scale cutoff of the theory under consideration (in other words, the micro-theory may be the directly 'underlying' the theory being studied, where the latter is called the macrotheory). ${ }^{20}$ Also, it is important to note that there are some examples where using

'a-mergence' or 'emergence+').

${ }^{18}$ The most basic characterisation of supervenience states that a set of properties $A$ supervenes upon another set $B$ just in case no two things can differ with respect to $A$-properties without also differing with respect to their $B$-properties (see, e.g. McLaughlin and Bennett (2011).)

${ }^{19}$ Although Butterfield (2011a) demonstrates that we can have emergence (with or) without supervenience, I believe that supervenience - as I have stated it here -holds in all of the cases I consider in this paper. Butterfield (2011a, §5.2.2) has two examples of emergence without supervenience. Briefly: the first example involves recognising the work of philosophers such as Silberstein and McGeever (1999), who present entangled quantum states as cases of emergence without supervenience. This involves a different notion of supervenience (mereological supervenience) than the one I've admitted here, and emergence is taken as a failure of supervenience. The second case that Butterfield (2011a) presents is counterfactual, involving the possibility of 'configurational forces'. Although the existence of such forces would pose a problem for utilising the idea of supervenience in my definition of emergence, we do not know of any such forces, and physics gives us no reason to suppose that such forces exist, so their metaphysical possibility does not affect my claims here.

${ }^{20} \mathrm{Or}$, of course, the micro-theory may be a theory however many levels up the tower from the theory of interest, depending on your interests and/or requirements. 
supervenience in order to define the comparison class in this way is not appropriate, for instance, in cases where the relevant comparison class should be defined without reference to the micro-theory.

By novelty, I mean that there are features of the macro-level that are not features of the micro-level, and cannot be exhibited by (or defined by) the micro-level. That is, there are striking differences between the two levels, each being described by different theories and different degrees of freedom. ${ }^{21}$ Novelty in the case of EFT comes from the fact that the low-energy theory is formally distinct from the high-energy theory - the degrees of freedom of the effective Lagrangian density are associated with states that are formally distinct from those associated with the degrees of freedom of the original (high-energy) Lagrangian density. This recognition forms the basis of both Zhang's (2004) and Bain's (2013b) conceptions of emergence in EFT.

In regard to autonomy, we may say that a particular level of the tower is autonomous from the one above it (i.e. the higher-energy theory underlying it) if, in addition to it being formally distinct from the latter, describing different physics and different degrees of freedom, it is impervious to changes in the high-energy system. $^{22}$ Usually there is not absolute autonomy, but rather quasi-autonomy, meaning that the level is independent of much or most of the high-energy physics. In the physical examples discussed here, autonomy stems from the high-energy theory being severely underdetermined by the low-energy physics. As we have seen, autonomy is very important, and its explanation certainly not trivial, in the literature on EFT, as shown above. Bain (2013) as well as Cao and Schweber (1993) tie their conceptions of emergence to autonomy in this sense.

The relevant sense of (quasi-) autonomy in EFT comes from the fact that the low-energy theory is largely independent of the details of the high-energy theory. As we have seen, the high-energy theory contains excess information far over-and-

\footnotetext{
${ }^{21}$ The term 'macro' is used only to contrast with 'micro' here: I mean these only as relative terms, where the former denotes the physics that is supposed to exhibit the 'Independence', while the latter is the relevant comparison class, with respect to which the 'macro' exhibits the requisite novelty and autonomy. I do not mean to imply that the emergent phenomena must always be confined to the macro-realm (we can say 'upper-level' or 'low-energy' to denote the same thing).

${ }^{22}$ My account is essentially similar to Butterfield's (2011a, p. 921) understanding of emergence as 'novel and robust behaviour' relative to some appropriate comparison class; but, as described above, my account differs in that it employs another tenet ('Dependence') which, in some cases, helps define the idea of a comparison class. Also, the idea of autonomy (robustness) is made concrete through the ideas of decoupling and underdetermination.
} 
above that required in order to describe the low-energy physics; this complexity is the reason for the difficulty in constructing EFTs. The low-energy physics actually depends on very little of the high-energy physics: some particular interactions and symmetries are important, but the details of the high-energy theory are not. This is the hallmark of the EFT program. The high-energy effects that filter down to low-energy are typically able to be realised by any number of different systems, and, as such, the high-energy theory is severely underdetermined by the low-energy physics. Additionally, there is a stronger sense of autonomy where the underdetermination is, in effect, 'physically realised', meaning that there are several different high-energy systems that give rise to the same low-energy physical behaviour. This is known as universality.

\section{Universality}

Universality is characterised as identical behaviour exhibited by many different physical systems.' It is essentially similar to the idea of multiple realisability, which is familiar in philosophy as the claim that any given macro-state can be realised by any one of a multiplicity of different micro-states. ${ }^{23}$ Typically, universality is used just to refer to those properties that an RG analysis is amenable to elucidating; this stipulation serves to exclude properties which are multiply-realised by virtue of being general, e.g. things like 'having mass' or 'being coloured', as counting as cases of universality. ${ }^{24}$ Universality provides a strong basis for autonomy: because a universal behaviour at low-energy can be realised by a number of different highenergy systems (systems which make up the 'universality class'), the low-energy behaviour must be autonomous from the details of the high-energy physics. In the next three sub-sections, I present three different examples of universality in physics.

\subsection{RG-fixed points and critical phenomena}

One example of universality is that of second-order phase transitions, where a number of different systems - different types of 'fluids', as well as magnetic materials exhibit the same critical phenomena. For many condensed matter systems, an IR fixed point (as mentioned in $\S 2$ ) corresponds to a second-order phase transition,

\footnotetext{
${ }^{23}$ This statement of multiple realisability comes from Fodor (1997).

${ }^{24}$ The ideas of multiple realisability, reduction and emergence have some history together in philosophy, particularly related to Kim's (1992) and Sober's (1999) responses to Putnam (1967; 1988 ) and Fodor $(1974 ; 1997)$. These arguments have been of great influence in the philosophy of mind, and concern the ideas of causality, scientific kinds, and reductionism in science. See Bickle (2008) for a concise presentation of the main arguments and replies.
} 
which is a transitions to a state which is an indistinguishable combination of two different states, or "phases", (e.g. a state where the distinction between liquid and vapour no longer makes sense for a fluid). The critical point for a system represents the conditions (e.g. the particular values of temperature and pressure) at which the system undergoes a second-order phase transition. At the critical point there is no distinguished length scale.

Critical behaviour is characterised by a value known as an order parameter (for fluids this is the difference in densities between the different coexisting phases). An order parameter is a quantity which is introduced in order to distinguish between two states of a system: its magnitude being zero in one phase and non-zero in the other. Generally, a non-zero order parameter corresponds to a state of broken symmetry (the zero value representing the symmetric state of the system), however, order parameters can be introduced in phase transitions not involving symmetrybreaking. For every fluid, the order parameter, $\Psi$, vanishes as some power, $\beta$, of $t$

$$
\Psi \propto t^{\beta}
$$

Where the reduced temperature, $t$, is defined in terms of the temperature $T$ and the critical temperature $T-c$, as, $t=\left|\left(T-T_{c}\right) / T_{c}\right|$. The reduced temperature shows how far from criticality a particular system is, and allows us to compare the critical behaviour of systems with different values of $T_{c}$.

$\beta$ is called a critical exponent. The fact that different systems exhibit the same behaviour as they approach $T_{c}$ (i.e. universality) is the statement that these systems all share the same value of $\beta$. The explanation for universality here comes from the $\mathrm{RG}$, and depends on the divergence of the correlation length, $\xi$, of the system. The correlation length is the average distance over which one microscopic variable is correlated with another. As the system approaches the critical temperature, the correlation length diverges: $\xi(T) \rightarrow \infty$ as $T \rightarrow T_{c}$. This means that near $T_{c}$ far distant points become correlated and long-wavelength fluctuations dominate. The range of influence of any one variable is extremely large, and a great number of degrees of freedom are coupled together. The RG is thus employed in order to make calculations tractable: knowing that the long-wavelength fluctuations are the ones of importance, we can integrate out the short-wavelength modes so that the large-scale behaviour is preserved. As the RG transformation is iterated, and the number of degrees of freedom reduced, we obtain a sequence of Hamiltonians that can usefully be pictured as defining a trajectory (i.e. RG "flow") in a space coordinatised by the system's parameters. These parameters include the temperature, the external fields, and the coupling constants. 
The correlation length remains infinite as further applications of the RG transformation are performed - in other words, at the critical point the system is invariant under the RG, indicating that $\infty$ is a fixed point. As Batterman (2011) is careful to point out, the fixed point should be understood as a property of the RG transformation itself, rather than a property of the system under consideration. ${ }^{25}$ Much of the physics near critical points can be understood by the transformations of the couplings. Performing a linearisation of the couplings in the neighbourhood of the fixed point on the "critical surface" (i.e. the surface defined by all those Hamiltonians with infinite correlation length) shows how the RG transformation acts upon points that differ only slightly from the fixed point itself. This analysis enables us to calculate the values of the critical exponents (recall that the critical exponents describe the behaviour of a system near the critical point) because it determines how lengths are scaled, locally, in different directions surrounding the fixed point. Such an analysis reveals that the fixed point has an associated basin of attraction, being all those points in the parameter space (the space of all possible Hamiltonians), which, under the RG transformation eventually flow into that fixed point. Now, universality is explained: systems with the same set of critical exponents lie in the basin of attraction of the same fixed point. ${ }^{26}$

The couplings of systems that belong to the same universality class are irrelevant, since they correspond to differences in these systems that ultimately have no impact on the behaviour of the systems at the critical point. The linearisation procedure allows us to classify the couplings of a system as irrelevant if they decrease under the RG transformation, and the system flows toward the fixed point. Conversely, the couplings are relevant if they increase under the RG transformation, and the system flows away from the fixed point, and marginal if their scaling behaviour is unable to be determined by the analysis. ${ }^{27}$ It is worth noting that the coincidence of the critical exponents across different types of systems was inexplicable prior to the development of the RG.

\section{$5.2 \quad$ Symmetry breaking}

The phenomenon of symmetry breaking furnishes another explanation of universality. It is related to the idea of a phase transition, as some phase transitions involve

\footnotetext{
${ }^{25}$ The relation between fixed points in the RG and critical phenomena was discovered by Wilson, building on the insights of Kadanoff. Michael Fisher, a colleague of Wilson's, also contributed greatly to our understanding in this area. See, for instance, Kadanoff (1966); Wilson $(1971,1974)$.

${ }^{26}$ This description is highly simplified. Please refer to Batterman (2011) and Pfeuty and Toulouse (1977, p. 12).

${ }^{27}$ See Butterfield and Bouatta (2012).
} 
symmetry breaking (and for a system to go from a symmetric to a non-symmetric state generally means the system has undergone a phase transition). An example of universality associated with symmetry breaking is found in superconductors (Morrison (2012) refers to this example in framing her account of emergence, as will be discussed shortly). There are universal properties that are common to all superconductors: infinite conductivity (currents that can circulate for years without perceptible decay, due to extremely low electrical resistance), flux quantization (the magnetic field is quantised in units of $h / 2 e$ ) and the Meissner effect (the expulsion of a magnetic field from a superconductor). ${ }^{28}$ These emergent features are said to be exact, meaning that they can be predicted with extraordinary accuracy.

As mentioned above, the prediction of the fine structure constant in precision tests of QED using the Josephson effect is one example of such a result. Recall that the BCS model used to obtain this result is actually an approximation, and the reason for its success is because it embodies a particular symmetry-breaking pattern. This is the same in the case of predicting exact results in superconductors - as Weinberg (1986, p. 43) states, the high-precision predictions about superconductors actually follow not from the details of the models themselves, but more generally from the fact that these models exhibit a spontaneous breakdown of a symmetry. Because they are exact results, Morrison (2012, p. 151) says, they must follow from general principles rather than the details of the approximations used to model them. This also agrees with Laughlin and Pine's (2000) claim that these properties of superconductors represent emergent phenomena.

While the BCS model provides a story about the micro-details of how superconductivity occurs, when it comes to deriving the universal characteristics of superconductivity, these micro-details are not essential in themselves. What is important is simply the breaking of the symmetry (electromagnetic gauge invariance) of the system. ${ }^{29}$ In this respect, the micro-story is significant only insofar as it serves to fill in the structure of the symmetry-breaking pattern. The high-energy details themselves are irrelevant, being severely underdetermined by the low-energy physics. According to the BCS model, while the system is in its symmetric (nonsuperconducting) state, above the critical temperature $T_{c}$, the electrons behave as free particles, repelled from one another because of their like charge.

\footnotetext{
${ }^{28}$ Note that there are other features of individual superconductors that are not universal: for instance, heat capacity and critical temperature (the temperature at and above which superconducting properties are no longer present) are dependent on the type of metal that the superconductor is composed of.

${ }^{29}$ The micro-details described by the BCS model are, however, used for approximate quantitative calculations, such as calculation of the critical temperature.
} 
At $T_{c}$ the electrons, on this model, form Cooper pairs - two electrons become correlated with one another, interacting via phonon exchange. When this occurs, the electromagnetic gauge invariance is broken and the system becomes superconducting, with its electromagnetic properties dominated by the Cooper pairs. The order parameter in this case is related to the macroscopic ground state wave function of the Cooper pairs, $\langle\varphi\rangle$. In the non-superconducting phase, $\langle\varphi\rangle=0$ since there are no Cooper pairs, while in the superconducting phase $\langle\varphi\rangle \neq 0$ since the Cooper pairs have formed. The nonzero order parameter indicates that the symmetry has been broken and a phase transition has occurred. The coherence length $\xi$ this case is the mean separation of electrons at which pair correlation becomes effective. ${ }^{30}$

As Weinberg (1986) explains, and Morrison (2012) re-emphasises, we do not need the story about the formation and behaviour of the Cooper pairs in order to derive the most important exact consequences of superconductivity. To do this, we need only the assumption of broken electromagnetic gauge invariance, and nothing depends on the specific mechanism by which the breakdown occurs. ${ }^{31}$ On this picture, $\varphi(x)$ is not taken to be related to the wavefunction of the Cooper pairs, as it is in the BCS model, but, rather, it is a Nambu-Goldstone field that accompanies the breakdown of electromagnetic gauge invariance. As Morrison (2012, p. 155) states, we do not need a micro-story of electron pairing: Planck's constant simply does not appear in the differential equation that govern $\varphi$.

It can be shown that infinite conductivity depends only on the spontaneous breakdown of electromagnetic gauge invariance. Although the micro-story of the BCS approximation provides a detailed mechanism about how this breakdown occurs, namely, by the formation of Cooper pairs, its details are not necessary for understanding infinite conductivity or the other important properties of superconductors - these being consequences of the broken symmetry. Importantly, this means that we could change the irrelevant details of the micro-story and still have superconductivity, with its characteristic properties, emerge. For instance, as Weinberg (1986, p. 49) points out, infinite conductivity would, presumably, occur even if the charged particles whose pairing resulted in the breakdown of the symmetry were bosons rather than fermions.

\footnotetext{
${ }^{30}$ The value of which is between 100 and 1000 nanometres, being three or four orders of magnitude larger than the lattice spacing.

${ }^{31}$ I will not recount the definitions of the relevant quantities and the derivations of the characteristic properties of superconductors here. See Morrison (2012); Weinberg (1986).
} 
We see that symmetry breaking furnishes a strong explanation of universality. Notice that any micro-description of a given superconductor, purely by virtue of being a particular micro-description, will fail to capture the universality of the phenomena. In this sense, we might say that the micro-story does not and cannot provide an account of the emergent (universal) phenomena. Laughlin and Pines (2000, p. 28) present symmetry breaking as an example of a "higher-order" organising principle, and the phenomena that depend on it are "transcendent" insensitive to, and unable to be deduced from, micro-details. As Morrison (2012, p. 149) states, too, the notion of symmetry breaking is not itself linked to any specific theoretical framework, rather, it functions as a structural constraint on many different kinds of systems, both in high-energy physics and condensed matter physics.

\section{Discussion: Emergence associated with uni- versality}

As the examples above demonstrate, universal features (described by low-energy effective theories) can be seen as novel and autonomous compared to the highenergy description of the system they emerge from. Each of the examples provides an explanation of universality, and it is best to understand them as different explanations rather than as being dependent on one another, or in some way complementary to one another. For instance, although a full description of the physics of critical phenomena involves both symmetry breaking and the mathematical apparatus of the RG, an explanation of the universality can be provided either by a story about symmetry breaking (where systems with the same symmetry breaking pattern exhibit the same behaviour) or by the RG (where systems in the same universality class lie in the basin of attraction of the same fixed point).

Although these are examples of EFTs, the ideas of novelty and autonomy afforded by instances of universality are different from those in EFT more generally. One important difference is that in the case of critical phenomena and symmetry breaking phase transitions, the relevant sense of emergence is diachronic: the features of the system following the phase transition are importantly novel compared to the system beforehand (when it was not at the conditions required for the phase transition to occur). In other examples of EFTs, the sense of emergence is better understood as synchronic: we compare the different levels at a given time, and there is no dynamical change in the system. 
Another important difference concerns the sense of autonomy applicable to these cases. Usually, the low-energy physics of an EFT is said to be quasi-autonomous from the high-energy physics, since it depends on only a few of the parameters that are important at low-energy (the high-energy theory being underdetermined by the low-energy physics). In cases of universality, however, there is a stronger sense in which the emergent theory may be said to be autonomous from the highenergy physics: we cannot account for the universality of the emergent behaviour across different systems by appeal to the high-energy details of any single system. In these cases, the explanation of the emergent behaviour comes not from EFT, but from the RG fixed-point story or symmetry breaking. Cases of universality are special instances of EFT where we have multiply-realised behaviour. In EFT more generally, the multiple-realisability is not always 'realised', meaning we may not possess real examples of physical systems exhibiting the same low-energy behaviour.

Batterman (2011) and Morrison (2012) both present accounts of emergence based on universality, but their accounts rely on different, more nuanced and exclusive definitions of emergence than the one presented here. These definitions are framed in terms of a failure of reduction, and, I think, suffer for it. As should be clear already, the focus on a failure of reduction is contrived and unnecessary. There are senses in which the low-energy physics can be reduced to the high-energy physics; the emergent behaviour supposedly can 'in principle' be derived from the high-energy description of the system using EFT (although, like Bain (2013a,b), we could argue that this does not count as a derivation), the RG and the idea of symmetry breaking. Of course, though, Batterman (2011) and Morrison (2012) are interested in universal behaviour because it is universal: reduction to any particular high-energy theory will fail to capture the universality (the fact that many other systems, as well as the one used in the derivation, exhibit the same behaviour). In other words: there may be reduction, but is not interesting.

The main problem with focusing on the reduction's failure to explain the universality is that it means we can never classify the behaviour of any single system as emergent. On these accounts, we need to have several examples of other physical systems with the same low-energy physics (but different high-energy physics) in order to say that there is emergence. It would be preferable, surely, to address a case of emergence without the need to consider additional, external systems. This is one of the benefits that my account offers. The positive conception of emergence as being simply the novelty and autonomy of the low-energy physics compared to the high-energy physics means we can consider individual cases of emergence. This definition of emergence is more general than those upheld by Batterman and Mor- 
rison, as it allows us to recognise emergence even in 'unrealised' cases of universal behaviour (i.e. cases where the emergent physics depends so minimally on the micro-theory that the latter could be any number of things, even when we do not have examples of these other high-energy systems).

Another problem with definitions of emergence based on a failure of reduction is that they are not truly 'physics-first', and so, by utilising them we risk overlooking the interesting relations in the physical examples. It is not necessary to start off with a definition of emergence that carries a lot of 'baggage': the physical relations are complicated enough as it is! As stated above, in $\S 3$, the intuition that emergence is a failure of reduction comes from a perceived need to classify cases of emergence as 'ontological' or 'merely explanatory', and is a hangover from debates in metaphysics and the philosophy of mind. Taking a definition of emergence based on reduction is more exclusive than the definition I propound here; however, Batterman and Morrison maintain that this restrictiveness is a positive aspect of their accounts, as it allows them to exclude things that they do not want to count as cases of emergence, and things that we would not intuitively count as cases of emergence. Yet, as I argue shortly, the comparatively general definition of emergence fits more naturally with the physics - the fact that it less resembles the conceptions of emergence in metaphysics is not a problem (so long as we are willing to admit different conceptions of emergence are applicable in different $\operatorname{cases}^{32}$ ).

Morrison's (2012, p. 143) definition of emergence comprises two statements:

Epistemological independence We need not appeal to micro-details in order to explain or predict the emergent phenomena

Ontological independence We cannot appeal to micro-details in order to explain or predict the emergent phenomena

While epistemological independence is necessary for emergence, Morrison says, only ontological independence is sufficient. The distinction is necessary, according to Morrison, in order to distinguish cases of emergence from low-energy behaviour that is "resultant" (where "emergent" behaviour is ontologically independent, but "resultant" behaviour is not). Morrison views epistemological independence as insufficient for emergence because "the fact that we need not appeal to micro phenomena to explain macro processes is a common feature of physical explanation across many systems and levels" (2012, p. 143). For Morrison, emergence requires complete autonomy of the macro (low-energy) level from the micro level: if there is

\footnotetext{
${ }^{32}$ And if you are not a pluralist when it comes to emergence, please refer to Footnote 17.
} 
an "ontological or explanatory link" between the two, then, Morrison says, we have "resultant" behaviour rather than emergence (p. 163). Of course, it is the idea of universality that allows for such a strong emergence claim: because the same low-energy behaviour can arise from systems that are very different at the microlevel, there is no obvious "ontological or explanatory link" between the micro and macro levels. If there was such a link, then we would have no explanation of how universality is possible.

There are two issues with Morrison's (2012) account of emergence that make the positive conception (expounded here) preferable to it. Firstly, there are difficulties with Morrison's (2012) account that result from its dependence upon universality and the issue mentioned above, that we cannot consider individual cases of emergence. It would seem, on such an account, that if we only had a single system (whose low-energy behaviour is described by an EFT), then we could say just that it exhibits 'epistemological independence' (using Morrison's definition, above) and not emergence. Although its low-energy properties depend only tenuously on the high-energy physics (and in such a way that we need not know the high-energy physics), Morrison would claim that these properties are still 'resultant' rather than emergent. Yet, as soon as another system, with different micro-composition but the same low-energy behaviour was discovered, the systems would both exhibit 'ontological independence' (using Morrison's definition) and demonstrate emergence. Suddenly, the micro-story becomes impotent: explanatory one minute, useless the next. Furthermore, it is especially problematic for Morrison's account of emergence, which claims to be an ontological account, that emergence depends on our state of knowledge.

Consider that if we had only a single example of a superconductor, composed of a particular type of metal, then we would not, according to Morrison's (2012) account, call its superconductivity an emergent phenomenon. The properties of the superconductor are derived using the BCS model, which tells an explanatory microstory about the formation of Cooper pairs at the critical temperature. We might recognise that the derivation of the superconductivity does not rely on the details of the micro-story but rather on the broken gauge symmetry alone, but this would not change our classification that the behaviour is resultant: after all, it is the micro-physics that actually exhibits ('fills in' or 'realises') the symmetry, and the fact that it does so is the reason we are able to recognise that the system exhibits the relevant pattern. Morrison (2012, pp. 162-163) argues that the symmetry breaking cannot be classified as fundamental physics (i.e. part of the micro-theory) and so the fact that superconductivity can be derived from it does not count relevantly as reduction. I do not want to argue about what counts as derivation or 
reduction, nor what should be classed as fundamental physics, but, in the absence of other examples of superconductors we cannot appeal to universality in order to recognise the superconductivity as emergent. Again, however, as soon as a different superconductor is discovered, the superconductivity becomes emergent on this account.

We can avoid these problems, of not being able to deal with individual cases of emergence, and having emergence depend on our knowledge of the existence of other systems, by adopting the more general, positive account of emergence I've expounded in this paper. Because it applies to all EFTs, rather than just those which happen to describe more than one low-energy system, the positive conception of emergence admits cases of 'potential' or 'unrealised' universality. It means that our ascription of emergence will not change once we discover that there are other systems with the same low-energy behaviour. This conception of emergence also fits more naturally with the physics by encompassing EFT generally (as applied), rather than a select group of EFTs (those which we happen to know apply to multiple systems). As stated above, Morrison (2012) rejects EFT and its 'epistemological independence' because it is common. I hope to have shown that this dismissal is hasty and disloyal to the physics - yes, the fact that we typically need not appeal to micro-details in order to explain macro-behaviour in science is certainly a pervasive one, but its great frequency and usefulness should make it more interesting, not less. It is a testimony to the power of the RG and EFT methods used in physics, and deserves attention; yet it is overlooked thanks to our fixation on reduction, derivation and ontology.

Regardless of this, however, if Morrison (2012) wishes to retain a more exclusive definition of emergence, yet avoid the 'individual case' problems, then less burden should be put on the idea of universality in her account. For example, Morrison could appeal to the fact that the characteristic properties of superconductors are exact results, and thus tie emergence to the "transcendent" higher-organising principles of Laughlin and Pines (2000). Not all instances of EFT produce exact results, but the examples involving symmetry breaking discussed above do. One reason for considering symmetry breaking as a higher-organising principle is that it is not linked to any specific theoretical framework; rather, as Morrison (2012) states, it functions as a "structural constraint" on many different kinds of systems in high-energy physics, condensed matter physics and cosmology. Mainwood (2006, p. 114) also makes this point, stating that, "Such principles [as symmetry breaking] that apply across physics of very different scales, give a sense of unity to physics, which implies no prejudice in favour of microphysics." 
Alternatively, Morrison could appeal to the fact that emergence associated with critical phenomena and symmetry breaking phase transitions is a diachronic sense of emergence, whereas in other cases of EFT the low-energy behaviour is novel and autonomous compared to the high-energy system at a given time. This is an important point that distinguishes Morrison's (2012) account, which emphasises the role of symmetry breaking in addition to the RG, from Batterman's (2012) account, which favours the RG fixed point story and dismisses the significance of symmetry breaking. Although the RG is said to induce a 'flow' in the parameters of a system, the RG not something that can properly be said to act - the parameters that 'flow' are those whose values are seen to differ as we change (by hand) the energy at which we view the system. The RG equations are simply a description. On the other hand, symmetry breaking is a process; it is a dynamical change in the state of the system.

Either of the two strategies immediately outlined would lessen the dependence of Morrison's (2012) account on the idea of universality, and, if developed, could preserve Morrison's exclusive, ontological account of emergence while guarding against the 'individual case' problems. Taking either of these strategies would exclude those cases of EFT that are not cases of universality, but also exclude some cases of universality that don't involve symmetry breaking - for instance, hydrodynamics and non-critical thermodynamics. As it currently stands, Morrison's (2012) account is not strong enough to distinguish between critical phenomena and these other cases, because all the work is done by the idea of universality. ${ }^{33}$

Here I should note that Batterman's (2011) account does not suffer from this problem; Batterman (2011) is able to make a distinction between the critical phenomena and hydrodynamics (and non-critical thermodynamics), as his definition of emergence requires there be a singularity. In the case of critical phenomena, the necessary singularity is the thermodynamic limit. This is the limit as the number of particles in a system approaches infinity, and is an essential idealisation invoked in accounting for phase transitions. Although, of course, the phase transition occurs in a finite system, the statistical mechanics of a finite number of particles cannot exhibit the non-analytic behaviour necessary to represent the qualitatively distinct phenomena that occur when the system undergoes a phase transition (Kadanoff , 2000, pp. 238-239). ${ }^{34}$ The thermodynamic limit is associated with the divergence of the correlation length as the system approaches the critical temperature, $\xi(T) \rightarrow \infty$ as $T \rightarrow T_{c}$. Batterman (2011) argues that this divergence is an essen-

\footnotetext{
${ }^{33}$ I.e. it is just the idea of universality that is used to make the distinction between 'epistemological independence' and 'ontological independence'.

${ }^{34}$ See also Butterfield and Bouatta (2012).
} 
tial aspect of the explanation of universality, an argument which succeeds only if we agree with Batterman that the RG fixed-point story is the correct (or, at least, the best) explanation of universality. The symmetry breaking account does not rely on the presence of a mathematical divergence.

The other difficulties with Morrison's (2012) conception of emergence are related to the fact that it is supposed to represent an ontological account of emergence. Presumably, Morrison wants to take the emergent properties as real, but her explanation of how they are arrived at employs both the RG fixed-point story as well as symmetry breaking. The RG fixed-point story involves an "unphysical" infinite idealisation, which we may not desire to be ontologically committed to. ${ }^{35}$ So, expounding an ontological account of emergence requires some indication of how we are to commit to our theories - why are we supposed to be committed to some parts of our theories and not others (even though they are apparently parts upon which the ontologically trustworthy parts rely)? The positive conception of emergence that I've presented in this paper, of course, avoids any such questions and holds that the development and criticism of realist positions is to be undertaken separately to the project of understanding emergence in physics.

\section{Conclusion}

Accepting pluralism in regards to emergence means accepting that there are many different conceptions of emergence, each of which may have its own domain of applicability. The conception of emergence espoused in this paper is presented as being one that is appropriate and important for understanding the relation between 'levels' of theories in physics. It has come from considering several physical examples, and in these examples the ideas of reduction and derivation are not usefully or interestingly related to emergence. The positive definition of emergence involves focusing on the novelty and autonomy of the low-energy physics compared to the high-energy description of a system. Novelty means that the low-energy theory is framed in terms of interactions that are not present at high-energy, it may be based on different symmetries, and describes behaviour that the highenergy theory is incapable of representing in such a way that we are able to grasp the relevant low-energy degrees of freedom. Autonomy, or quasi-autonomy, of the low-energy theory is due to the fact that, typically, the low-energy physics depends only tenuously on the high-energy physics - the link being so minimal

\footnotetext{
${ }^{35}$ There may also difficulties with naive realism when it comes to trusting our theories of spontaneous gauge symmetry breaking, see Elitzur (1975) and the recent response Friedrich (2013).
} 
that any number of high-energy theories could do the job. In other words, the low-energy physics underdetermines the high-energy theory.

In cases of universality, we actually have examples of systems that differ at high-energy, yet exhibit the same characteristic low-energy behaviour. There are two other accounts of emergence that are based on universality (viz. Batterman (2011) and Morrison (2012), but I have argued that the positive conception outlined in this paper is preferable since it considers the physics as practised and is not weighed-down by difficulties related to an unnecessary focus on reduction, derivation and ontology. While this positive conception of emergence may not appeal to philosophers who take emergence to be defined as a failure of reduction (in some sense), I hope that this paper will draw attention to the overlooked relations in physics.

\section{Acknowledgements}

Thank you to Robert Batterman and Ian McKay for their comments on a previous draft of this paper. I am appreciative, also, for the suggestions made by the anonymous reviewers. While writing this paper, I was supported by funding from the John Templeton Foundation.

\section{References}

Anderson, Philip W. 1972. More is different. Science, 177: 393-396.

Appelquist, Thomas, and James Carazzone. 1975. Infrared singularities and massive fields. Physical Review D, 11: 2856-2861.

Bain, Jonathan. 2013a. Effective field theories. In The Oxford handbook of philosophy of physics ed. Robert Batterman, 224-254. New York: Oxford University Press.

Callender, Craig. 2013. Turn and face the strange... ch-ch-changes: philosophical questions raised by phase transitions. In The Oxford handbook of philosophy of physics ed. Robert Batterman, 189-223. New York: Oxford University Press.

Bain, Jonathan. 2013b. Emergence in effective field theories. European journal for philosophy of science, 3: 257-273.

Batterman, Robert W. 2011. Emergence, singularities, and symmetry breaking. Foundations of physics, 41: 1031-1050. 
Bazavov, Alexei, Claude Bernard, Carleton DeTar, Steven Gottlieb, U.M. Heller, J.M. Hetrick, J. Laiho, M. Levkova, P.B. Mackenzie, M.B. Oktay, R. Sugar, D. Toussaint, and R.M. Van de Water. 2010. Nonperturbative QCD simulations with $2+1$ flavors of improved staggered quarks. Reviews of modern physics, 82: $1349-1417$.

Bedau, Mark. A. 1997. Weak emergence. In Philosophical perspectives: Mind, causation, and world, vol. 11. ed. James E. Tomberlin, 375-399. Oxford: Blackwell Publishers.

Bickle, John. 2008. Multiple realizability. The stanford encyclopedia of philosophy. http://plato.stanford.edu/archives/fall2008/entries/multiple-realizability/ Accessed 23 February 2013.

Burgess, Cliff P. 2004. Quantum gravity in everyday life: General relativity as an effective field theory. Living reviews in relativity. www.livingreviews.org/lrr2004-5/ Accessed 23 February 2013.

Butterfield, Jeremy. 2011a. Emergence, reduction and supervenience: A varied landscape. Foundations of physics, 41: 920-959.

Butterfield, Jeremy. 2011b. Less is different: Emergence and reduction reconciled. Foundations of physics, 41: 1065-1135.

Butterfield, Jeremy, and Nazim Bouatta. 2012. Emergence and reduction combined in phase transitions. Proceedings of frontiers of fundamental physics 11, 1446: 383-403.

Butterfield, Jeremy, and Chris Isham. 1999. On the emergence of time in quantum gravity. In The arguments of time, ed. Jeremy Butterfield, 116-168. Oxford: Oxford University Press.

Callaway, David J. E., and Aneesur Rahman. 1982. Microcanonical ensemble formulation of lattice gauge theory. Physical review letters, 49: 613-616.

Callaway, David J. E., and Aneesur Rahman. 1983. Lattice gauge theory in the microcanonical ensemble. Physical review d, 28: 1506-1514.

Cao, Tian Yu, and Silvan Schweber. 1993. The conceptual foundations and the philosophical aspects of renormalization theory. Synthese, 97: 33-108.

Castellani, Elena. 2002. Reductionism, emergence, and effective field theories. Studies in history and philosophy of modern physics, 33: 251-267. 
Dürr, Stephan, Zoltán Fodor, Julien Frison, C. Hoelbling, R. Hoffmann, S.D. Katz, S. Krieg, T. Kurth, L. Lellouch, T. Lippert, K. Szabo, and G. Vulvert. 2008. Ab initio determination of light hadron masses. Science, 322: 1224-1227.

Elitzur, Shmuel. 1975. Impossibility of spontaneously breaking local symmetries. Physical review d, 12: 3978-2982.

Fodor, Jerry. 1974. Special sciences: Or the disunity of science as a working hypothesis. Synthese, 28: 97-115.

Fodor, Jerry. 1997. Special sciences: Still autonomous after all these years. Tomberlin, 149-164.

Friedrich, Simon. 2013. Gauge symmetry breaking in gauge theories: in search of clarification. European journal for philosophy of science, 3: 157-182.

Georgi, Howard. 1993. Effective field theory. Annual review of nuclear and particle science, 43: 209-252.

Hartmann, Stephan. 2001. Effective field theories, reductionism and scientific explanation. Studies in history and philosophy of modern physics, 32: 267-301.

$\mathrm{Hu}$, Bei-Lok. 2005. Can spacetime be a condensate? International journal of theoretical physics, 44: 1785-1806.

Hu, Bei-Lok. 2009. Emergent/quantum gravity: macro/micro structures of spacetime. In Fourth international workshop dice 2008: From quantum mechanics through complexity to spacetime: The role of emergent dynamical structures. Journal of Physics Conference Series, vol. 174. eds. H.T. Elze, L. Diosi, L. Fronzoni, J. Halliwell, and G. Vitiello, 12015. Bristol: Iop Publishing Ltd.

Huggett, Nick, and Robert Weingard. 1995. The renormalisation group and effective field theories. Synthese, 102: 171-194.

Kadanoff, Leo. 1966. Scaling laws for Ising models near $T_{c}$. Physics, 2: 263-272.

Kadanoff, Leo. 2000. Statistical physics: Statics, dynamics and renormalization. Singapore: World Scientific.

Kim, Jaegwon. 1992. Multiple realization and the metaphysics of reduction. Philosophy and phenomenological research, 52: 1-26.

Laughlin, Robert B., and David Pines. 2000. The theory of everything. Proceedings of the national academy of sciences of the United States of America, 97: 28-31. 
Lepage, Peter. 1989. What is renormalization? In From actions to answers, proceedings of the 1989 theoretical study institute in elementary particle physics. eds. T. Toussaint, and D. DeGrand, 483-509. Singapore: World Scientific.

McLaughlin, Brian, and Karen Bennett. 2011. Supervenience. The stanford encyclopedia of philosophy. http://plato.stanford.edu/archives/win2011/entries/supervenience/ Accessed 20 February 2013.

Morrison, Margaret. 2012. Emergent physics and micro-ontology. Philosophy of science, 79: 141-166.

Nambu, Yoichiro, and Giovanni Jona-Lasinio. 1961a. Dynamical model of elementary particles based on an analogy with superconductivity. i. Physical review, 122: $345-358$.

Nambu, Yoichiro, and Giovanni Jona-Lasinio. 1961b. Dynamical model of elementary particles based on an analogy with superconductivity. ii. Physical review, 124: $246-254$.

Pfeuty, Pierre, and Gerard Toulouse. 1977. Introduction to the renormalization group and to critical phenomena. London: Wiley.

Pich, Antionio. 1998. Effective field theory. arXiv http://arxiv.org/abs/hepph/9806303v1/ Accessed 17 February 2011.

Polchinski, Joseph. 1993. Effective field theory and the Fermi surface. In Proceedings of the 1992 Theoretical Advanced Studies Institute in Elementary Particle Physics. eds. Jeffrey Harvey and Joseph Polchinski. Singapore: World Scientific.

Putnam, Hilary. 1967. Psychological predicates. In Art, mind, and religion. eds. W.H. Capitan and D.D. Merrill, 37-48. Pittsburgh: University of Pittsburgh Press.

Putnam, Hilary. 1988. Representation and reality. Cambridge, MA: MIT Press.

Robinson, Don. 1992. Renormalization and the effective field theory programme. PSA: Proceedings of the biennial meeting of the philosophy of science association 1992, 393-403.

Shifman, Mikhail. 1998. Snapshot of hadrons. Progress of theoretical physics supplement, 131: 1-71.

Silberstein, Michael. 2012. Emergence and reduction in context: Philosophy of science and/or analytic metaphysics. Metascience, 21: 627-642. 
Silberstein, Michael, and John McGeever. 1999. The search for ontological emergence. The philosophical quarterly, 49: 182-200.

Sober, Elliott. 1999. The multiple realizability argument against reductionism. Philosophy of science, 66: 542-564.

Weinberg, Steven. 1986. Superconductivity for particular theorists. Progress of theoretical physics supplement, 86: 43-53.

Wilson, Kenneth G. 1971. The renormalization group (RG) and critical phenomena 1. Physical review b, 4: 3174 .

Wilson, Kenneth G., and John Kogut. 1974. The renormalisation group and the $\epsilon$ expansion. Physics reports, 12: 75-199.

Zee, Anthony. 2010. Quantum field theory in a nutshell. Second edn. Princeton: Princeton University Press.

Zhang, Soucheng. 2004. To see a world in a grain of sand. In Science and ultimate reality: quantum theory, cosmology, and complexity. eds. John D. Barrow, Paul C.W. Davies, and Charles L. Harper. Cambridge: Cambridge University Press. 